\title{
Efficacy and Risk Factors Associated to Resistance to Single-Agent Chemotherapy in Low-Risk Gestational Trophoblastic Neoplasia
}

\author{
Mamour Gueye*, Mame Diarra Ndiaye-Gueye, Serigne Modou Kane-Gueye, Fatou Niass Dia, \\ Aissatou Thiam, Sr. Elisabeth Diouf, Jean Charles Moreau \\ Gynecologic and Obstetric Clinic, Aristide Le Dantec Teaching Hospital, Cheikh Anta Diop University, Dakar, \\ Senegal \\ Email: "mamourmb@yahoo.fr
}

Received 10 December 2015; accepted 18 January 2016; published 21 January 2016

Copyright (C) 2016 by authors and Scientific Research Publishing Inc. This work is licensed under the Creative Commons Attribution International License (CC BY). http://creativecommons.org/licenses/by/4.0/

(c) (i) Open Access

\section{Abstract}

Objectives: This study aimed to assess efficacy of intramuscular methotrexate 8-day protocol in the treatment of low-risk gestational trophoblastic neoplasia and also identify prognostic factors associated with treatment failure, necessitating second line chemotherapy. Methods: This study was performed at Gynaecologic and Obstetric Clinic of Dakar Teaching Hospital, the reference Centre of Gestational Trophoblastic Disease in Senegal. At the beginning of 2011, patients were followed according to FIGO's recommendations. From 2011 to 2014, we diagnosed 88 low-risk gestational trophoblastic neoplasia (GTN) patients (WHO score < 7). Low-risk patients started their treatment with methotrexate (MTX) based on the 8-day protocol consisting of $1 \mathrm{mg} / \mathrm{kg}$ MTX in combination with $0.1 \mathrm{mg} / \mathrm{kg}$ folinic acid (FA) every other day. Resistance to treatment was the main outcome. We studied the association of different prognostic factors included in the World Health Organisation (WHO) scoring system and resistance to the initial single agent chemotherapy. Results: Eighty-eight patients were diagnosed for GTN during the study period. Average age was 31 years. The antecedent pregnancy was molar in $\mathbf{9 8 . 1 \%}$ of cases. Seventy-four patients underwent remission after single agent-chemotherapy. Resistance rate to single-agent chemotherapy was $15.9 \%$ (14 patients). Nine of them achieved remission after second line chemotherapy. WHO score was significantly associated with the risk of resistance to single-agent chemotherapy. Other variables included in the WHO as age, antecedent pregnancy, pre-treatment hCG, tumour size and FIGO stage were not significantly associated with resistance. We report five fatal cases. Conclusion: The 8-day protocol consisting of $1 \mathrm{mg} / \mathrm{kg}$ MTX in combination with $0.1 \mathrm{mg} / \mathrm{kg}$ folinic acid (FA) every other day is effective for women with LRGTN. The only significant prognostic factor for fail"Corresponding author. 
ure is pretreatment WHO score. We highly recommend the use of this protocol particularly in developing countries where methotrexate is available, affordable and relatively safe.

\section{Keywords}

\section{Gestational Trophoblastic Neoplasia, Chemotherapy, International Federation of Gynecology and Obstetrics, Resistance}

\section{Introduction}

The gestational trophoblastic disease (GTD) is a disease of the proliferative trophoblastic allograft that consists of: partial mole; complete hydatidiform mole; invasive and metastatic mole; choriocarcinoma; placental-site trophoblastic tumour (PSTT); and epithelioid trophoblastic tumour (ETT) [1]. After uterine evacuation, 80\% of hydatiform mole cases follow spontaneous remission, but in about $20 \%$ of cases, trophoblastic tissue escapes maternal immunological surveillance, invades the maternal host, and progresses to postmolar gestational trophoblastic neoplasm (GTN) [2].

In 2002, the International Federation of Gynaecology and Obstetrics (FIGO) and the World Health Organization (WHO), combined anatomic staging with a modification of the WHO prognostic index score, for an internationally agreed risk assessment and treatment approach (Table 1 and Table 2) [1]. While in the traditional WHO scoring system the GTN was divided into low, medium and high-risk groups, in the new system the GTN is stratified into two groups: 1) low-risk group (score $\leq 6$ ) intimating single-agent chemotherapy; and 2) highrisk group (score $>6$ ) mandating multi-agent chemotherapy [1].

Single-agent chemotherapy with sequential methotrexate (MTX)/actinomycin D (ActD) is the preferred treatment in patients with stage I GTN who desire to retain fertility and in patients with low-risk metastatic GTN with a rate of response of $83.5 \%$ of patients with stage I GTN, $80 \%$ patients with low-risk stage II GTN and 81.8\% patients with low-risk stage III GTN [2].

GTN patients, who present with a low-risk score, respond then well to chemotherapy. However, an appropriate assessment of the risk of failure with single agent therapy and selection of the appropriate treatment is essential. In low-income counties, MTX is more available than other drugs and is used for treatment of low-risk gestational trophoblastic neoplasia.

This study aimed to assess efficacy of intramuscular methotrexate 8-day protocol in the treatment of low-risk

\begin{tabular}{cl} 
Table 1. FIGO anatomical staging [1]. \\
\hline Stage I & Disease confined to the uterus \\
Stage II & GTN extends outside of the uterus, but is limited to the genital structures (adnexa, vagina, broad ligament) \\
Stage III & GTN extends to the lungs, with or without known genital tract involvement \\
Stage IV & All other metastatic sites
\end{tabular}

Table 2. Modified WHO prognostic scoring system as adapted by FIGO [1].

\begin{tabular}{|c|c|c|c|c|}
\hline Scores & $\mathbf{0}$ & 1 & 2 & 4 \\
\hline Age (years) & $<40$ & $\geq 40$ & & \\
\hline Antecedent pregnancy & Mole & Abortion & Term & \\
\hline Antecedent pregnancy from index pregnancy & $<4$ & $4-6$ & $7-12$ & $\geq 13$ \\
\hline Pre-treatment serum hCG (UI/ml) & $<10^{3}$ & $<10,000$ & $<100,000$ & $\geq 10^{5}$ \\
\hline Largest tumor size (including uterus) & & $3-<5 \mathrm{~cm}$ & $\geq 5 \mathrm{~cm}$ & \\
\hline Site of metastasis & Lung & Spleen, kidney & Gastro-intestinal & Liver, Brain \\
\hline Number of metastases & 0 & $1-4$ & $5-8$ & $>8$ \\
\hline Previous failed chemotherapy & & & Single drug & 2 or more drugs \\
\hline
\end{tabular}


gestational trophoblastic neoplasia (LRGTN) and also identify prognostic factors associated with treatment failure, necessitating second line chemotherapy.

\section{Methods}

This study was performed at Gynaecologic and Obstetric Clinic of Dakar Teaching Hospital, the reference Centre of GTD in Senegal.

Two protocols of follow up were used in our centre in two points of time. From 2006 to 2011, women were followed-up in accordance with hospital protocol called "Score de Dakar" [3]. This score includes age, parity, area of residence, blood group, revenue, level of post-evacuation hCG, character of vesicles, duration of pregnancy and history of hydatiform mole. According to this score, patients were classified into low (LR), medium (MR) and high risk (HR) of progression to GTN. For the LR, monitoring of hCG is recommended, for MR, single agent chemotherapy (Chem-P) with methotrexate (25 mg per day from D1 to D5) is prescribed and for HR patients, hysterectomy associated to chemotherapy (Chem-T) (methotrexate $20 \mathrm{mg} / \mathrm{Kg}$ i.m and cyclophosphamide $500 \mathrm{mg}$ i.v: day 1 and day every 3 weeks) is recommended until achieving undetectable serum hCG.

At the beginning of 2011, patients were followed according to FIGO's recommendations.

From 2011 to 2014, we diagnosed 88 low-risk gestational trophoblastic neoplasia (GTN) patients.

FIGO 2002 criteria were used to diagnose post-molar GTN: a high $\beta$-hCG level that has remained stable for four consecutive tests in 3 weeks, a 10\% increase in $\beta$-hCG level for three consecutive tests in 2 weeks, any elevation in $\beta$-hCG level for 6 months after evacuation, a histopathologic diagnosis of choriocarcinoma.

Staging and scoring GTN patients were done according to Table 1 and Table 2.

Patients of FIGO score less than 7 were considered to be low risk and included in this study. In our hospital, low-risk patients start their treatment with methotrexate (MTX) based on the 8-day protocol consisting of 1 $\mathrm{mg} / \mathrm{kg}$ MTX in combination with $0.1 \mathrm{mg} / \mathrm{kg}$ folinic acid (FA) every other day.

Stable or increasing $\beta$-hCG levels after two courses of treatment were considered a failure to the initial single-agent chemotherapy. Patients were switched to a combination therapy with EMA-CO (etoposide, methotrexate, actinomycin D, cyclophosphamide, oncovin) or MAC (methotrexate, actinomycin D, cyclophosphamide) or other combinations according to availability of drugs and means of patients.

We used resistance to single-agent chemotherapy as the primary outcome and studied the frequency of resistance among low-risk GTN patients.

The patients with resistance to single-agent chemotherapy (cases) were compared with the patients who responded to chemotherapy (controls). Because the WHO scoring system is based on the values assigned to the different prognostic factors, the FIGO score was used only in univariate analysis. SPSS (Statistical Package for Social Science, 20.0) software was used for statistical analyses.

\section{Results}

Eighty-eight patients were diagnosed for GTN during the study period. Average age was 31 years (14 - 54). The antecedent pregnancy was molar in $98.1 \%$ of cases while we observed one full term pregnancy. In $60.4 \%$ of patients no tumour was reported.

Ten patients (12.5\%) underwent hysterectomy for GTN treatment.

Patient characteristics are presented in Table 3.

Seventy-four patients underwent remission after single agent-chemotherapy. Mean number of MTX chemotherapy cycles required to achieve complete response was 6.9 which included administration of two additional cycles past initial normalization of $\beta$-hCG titers (range 2 - 23 cycles).

Resistance rate to single-agent chemotherapy was $15.9 \%$ (14 patients). Nine of them achieved remission after multi-agent chemotherapy (Etoposide-Vincristine-Cyclophosphamide, 5FU-MTX-Etoposide, EMACO, MTXAct-D-Cyclophosphamide). Finally, 83 patients underwent remission (94.3\%).

WHO score was significantly associated with the risk of resistance to single-agent chemotherapy (0.03). Other variables included in the WHO score as age, antecedent pregnancy, pre-treatment hCG, tumour size and FIGO stage were not significantly associated with resistance (Table 4).

Pre-treatment serum hCG $>100,000 \mathrm{IU} / \mathrm{L}$ was found in 9 patients (2 in the group with resistance) whereas only 6 patients had stage II or III (2 in the group with resistance).

We report five fatal cases. As they experienced resistance, multi-agent chemotherapy was prescribed. Unable 
Table 3. Patient characteristics.

\begin{tabular}{|c|c|c|}
\hline Variables & Number of patients (n) & Percentage (\%) \\
\hline \multicolumn{3}{|l|}{ Age range (years) } \\
\hline$<40$ & 69 & 78.4 \\
\hline$\geq 40$ & 19 & 21.6 \\
\hline \multicolumn{3}{|c|}{ Antecedent pregnancy } \\
\hline Molar & 87 & 98.9 \\
\hline Term & 2 & 1.1 \\
\hline \multicolumn{3}{|c|}{ Interval from index pregnancy (months) } \\
\hline$<4$ & 46 & 52.3 \\
\hline$\geq 4$ & 42 & 47.7 \\
\hline \multicolumn{3}{|c|}{ Largest tumour size in $\mathrm{cm}$} \\
\hline 0 & 60 & 68.2 \\
\hline$<3$ & 28 & 31.8 \\
\hline \multicolumn{3}{|c|}{ Pre-treatment serum hCG level (UI/ml) } \\
\hline$<100,000$ & 79 & 89.8 \\
\hline$\geq 100,000$ & 9 & 10.2 \\
\hline \multicolumn{3}{|l|}{ FIGO stage } \\
\hline I & 82 & 93.2 \\
\hline II & 6 & 6.8 \\
\hline III & 3 & 2,1 \\
\hline \multicolumn{3}{|l|}{ Risk score } \\
\hline$<4$ & 60 & 68.2 \\
\hline$\geq 4$ & 28 & 31.8 \\
\hline
\end{tabular}

Table 4. Comparison of groups by outcome.

\begin{tabular}{|c|c|c|c|c|}
\hline Variables & Number of patients with remission n (\%) & Number of patients with resistance $n(\%)$ & Total & p value \\
\hline \multicolumn{5}{|c|}{ Age range (years) } \\
\hline$<40$ & $60(87)$ & $9(13)$ & $69(78.4)$ & \multirow{2}{*}{0.1} \\
\hline$\geq 40$ & $14(73.7)$ & $5(26.3)$ & $19(21.6)$ & \\
\hline \multicolumn{5}{|c|}{ Interval from index pregnancy (months) } \\
\hline$<4$ & $39(84.8)$ & $7(15.2)$ & $46(52.3)$ & \\
\hline$\geq 4$ & 35 (83.3) & $7(16.7)$ & $42(47.7)$ & \\
\hline \multicolumn{5}{|c|}{ Largest tumour size in $\mathbf{c m}$} \\
\hline 0 & $51(85)$ & $9(15)$ & $60(68.2)$ & \multirow{2}{*}{0.4} \\
\hline$<3$ & $23(82.1)$ & $5(17.9)$ & $28(31.8)$ & \\
\hline \multicolumn{5}{|c|}{ Pre-treatment serum hCG level (UI/ml) } \\
\hline$<100,000$ & $67(84.8)$ & $12(15.2)$ & 79 (89.8) & \multirow{2}{*}{0.47} \\
\hline$\geq 100,000$ & $7(77.8)$ & $2(22.2)$ & $9(10.2)$ & \\
\hline \multicolumn{5}{|l|}{ FIGO stage } \\
\hline I & $70(85.4)$ & $12(14.6)$ & $82(93.2)$ & \multirow{2}{*}{0.2} \\
\hline II-III & $4(66.7)$ & $2(33.3)$ & $6(6.8)$ & \\
\hline \multicolumn{5}{|c|}{ WHO score } \\
\hline$<4$ & $54(90)$ & $6(10)$ & $60(68.2)$ & \multirow{2}{*}{0.031} \\
\hline$\geq 4$ & $20(71.4)$ & $8(28.6)$ & $28(31.8)$ & \\
\hline \multicolumn{5}{|c|}{ Surgery before treatment } \\
\hline Yes & $5(50)$ & $5(50)$ & $10(11.4)$ & \multirow{2}{*}{0.008} \\
\hline No & $69(88.5)$ & $9(11.5)$ & $78(88.6)$ & \\
\hline
\end{tabular}


to achieve the chemotherapy drugs appropriate to their condition (MAC or EMACO), they gave up and return to their village. After a couple of weeks, they were readmitted with visceral metastases and deceased in the following days.

\section{Discussion}

Resistance to single agent treatment was significantly associated with a FIGO score higher than 4 but not to other variables of the WHO score as interval from the index pregnancy, $\beta$-hCG level higher than 100,000 IU/L.

We encounter several difficulties in diagnosis and management of gestational trophoblastic neoplasia. Monitoring of hCG is not properly achieved by all patients. Once the diagnosis of NTG established, the initiation of chemotherapy may be delayed for financial reasons. In case of resistance, a second-line chemotherapy is not automatically established. These are the limitations of this study.

Extensive experience has been accumulated in treating low-risk GTN over time, and over 14 different types of chemotherapy regimens have been described, but no consensus has been reached about the preferred first-line treatment. As there is no strong evidence to confirm the superiority of any one method, several treatments have been arbitrarily used in different centres. However, a consensus has been reached about the use of a single agent, such as MTX or ActD, for patients with low-risk disease. These drugs have induction remission rates of $50 \%$ to $90 \%[4]$.

Primary response variability results from differences in drug doses, times and administration modes, as well as patient selection. In general, weekly IM or intermittent IV infusion of MTX and biweekly ActD are less effective than MTX and ActD for five days or MTX/FA for eight days. However, almost all patients are cured and have their fertility preserved despite the differences in initial remission after primary chemotherapy [4]. The weekly MTX 30 - $50 \mathrm{mg} / \mathrm{m}^{2}$ regimen has the advantage of convenience and low cost and toxicity, but has the lowest complete response rate among all other regimens [4].

With methotrexate associated or not to folinic acid for eight days, rate of response was near $95 \%$ in our study.

In The Cochrane review, Alazzam et al. aimed to determine the efficacy and safety of first line chemotherapy in the treatment of low risk GTN [5]. The review included randomised controlled trials (RCTs) [6] [7], quasi-RCTs [8]-[10] and non-RCTs (cohort and case control studies (CCS) [11] [12]) for the treatment of low risk GTN. Eight studies met the review entry criteria $(n=769)$. Based on the available evidence from the included RCTs, the authors conclude that "pulsed" dactinomycin is superior to weekly parenteral methotrexate at the reported dosages. However, the authors believe that rigorously designed, multicentred, randomised double-blind trials are required to evaluate other combinations of chemotherapy regimens, most importantly "pulsed" dactinomycin with the widely used 8-day methotrexate-folinic acid [5].

Finally, 8-day methotrexate-folinic acid is effective in treatment of low risk gestational trophoblastic neoplasia. However, clinical trials are needed to find out affordable schedules or drugs for a better treatment of this intermediate group. In spite of different management and treatment approaches in GTN patients and regardless of their initial response, all the patients with a low-risk disease ultimately achieved remission even if second-line regimens are very difficult to implement in our context. Act D used in MAC and EMACO protocols is often obtained through donations. This drug whose effectiveness is recognized is not always available in our country. Moreover, its high price limits its access.

In developing world cost of treatment remains a major concern. MTX is a relatively safe, effective and inexpensive drug and thereby more easily available to Senegalese patients.

\section{Conclusion}

The 8-day protocol consisting of $1 \mathrm{mg} / \mathrm{kg}$ MTX in combination with $0.1 \mathrm{mg} / \mathrm{kg}$ folinic acid (FA) every other day is effective for women with LRGTN. The only significant prognostic factor for failure is pretreatment WHO score. We highly recommend the use of this protocol particularly in developing countries where methotrexate is available, affordable and relatively safe.

\section{References}

[1] FIGO Oncology Committee (2002) FIGO Staging for Gestational Trophoblastic Neoplasia. International Journal of Gynecology and Obstetrics, 77, 285-287. http://dx.doi.org/10.1016/S0020-7292(02)00063-2

[2] Berkowitz, R.S. and Goldstein, D.P. (2009) Current Management of Gestational Trophoblastic Diseases. Gynecologic 
Oncology, 112, 654-662. http://dx.doi.org/10.1016/j.ygyno.2008.09.005

[3] Cissé, C.T., Sall, A., Moreau, J.C., et al. (2005) Prévention du choricarcinome post-môlaire en milieu africain: exemple du Sénégal. La Lette du Gynécologue, 307, 8-14.

[4] Biscaro, A., Braga, A. and Berkowitz, R.S. (2015) Diagnosis, Classification and Treatment of Gestational Trophoblastic Neoplasia. Revista Brasileira de Ginecologia e Obstetrícia, 37, 42-51. http://dx.doi.org/10.1590/SO100-720320140005198

[5] Alazzam, M., Tidy, J., Hancock, B.W., et al. (2009) First Line Chemotherapy in Low Risk Gestational Trophoblastic Neoplasia (Review). The Cochrane Collaboration, 1, 1-11. http://dx.doi.org/10.1002/14651858.cd007102.pub2

[6] Osborne, R., Filiaci, V., Schink, J., et al. (2008) A Randomized Phase III Trial Comparing Weekly Parenteral Methotrexate and "Pulsed" Dactinomycin as Primary Management for Low Risk Gestational Trophoblastic Neoplasia: A Gynecologic Oncology Group Study. Gynecologic Oncology, 108, S2-S31.

[7] Lertkhachonsuk, A., Tangtrakul, S., Israngura, N., et al. (2009) Actinomycin d versus Methotrexate-Folinic Acid as the Treatment of Stage I, Low-Risk Gestational Trophoblastic Neoplasia: A Randomized Controlled Trial. International Journal of Gynecological Cancer, 19, 985-988. http://dx.doi.org/10.1111/IGC.0b013e3181a8333d

[8] Yarandi, F., Eftekhar, Z., Shojaei, H., et al. (2008) Pulse Methotrexate versus Pulse Actinomycin D in the Treatment of Low-Risk Gestational Trophoblastic Neoplasia. International Journal of Gynecology and Obstetrics, 103, 33-37. http://dx.doi.org/10.1016/j.ijgo.2008.05.013

[9] Gilani, M.M., Yarandi, F., Eftekhar, Z., et al. (2005) Comparison of Pulse Methotrexate and Pulse Dactinomycin in the Treatment of Low-Risk Gestational Trophoblastic Neoplasia. Australian and New Zealand Journal of Obstetrics and Gynaecology, 45, 161-164. http://dx.doi.org/10.1111/j.1479-828X.2005.00366.x

[10] Abrao, R.A., de Andrade, J.M., Tiezzi, D.G., et al. (2008) Treatment for Low-Risk Gestational Trophoblastic Disease: Comparison of Single-Agent Methotrexate, Dactinomycin and Combination Regimens. Gynecologic Oncology, 108, 149-153. http://dx.doi.org/10.1016/j.ygyno.2007.09.006

[11] Smith, H.O. and Kim, S.J. (2003) Epidemiology. In: Hancock, B.W., Newlands, E.S., Berkwitz, R.S. and Cole, L.A., Eds., Gestational Trophoblastic Diseases, 2nd Edition, International Society for the Study of Trophoblastic Diseases, 39-76.

[12] Wong, L.C., Choo, Y.C. and Ma, H.K. (1984) Oral Etoposide in Gestational Trophoblastic Disease. Cancer Treatment Reports, 68, 775-777. 